\title{
Las nanotecnologías en la crisis mundial
}

\section{Guillermo Foladori* Noela Invernizzi ${ }^{* *}$}

\begin{abstract}
Resumen: El artículo analiza el desarrollo de las nanotecnologías en el contexto de la irrupción de la crisis económica mundial. Comienza destacando los elementos claves de una revolución tecnológica: las características técnicas, el papel en la acumulación de capital y el impacto sobre las clases sociales. Luego se analiza el lugar de la tecnología en el desarrollo, para terminar mostrando cómo las tendencias a la concentración del capital están envolviendo a las nanotecnologías, lo cual puede llevar a un mundo más desigual al que ya existe hoy en día. Las nanotecnologías constituyen la base de la próxima revolución industrial que ya se está estableciendo. Con la irrupción de la crisis económica mundial también las nuevas tecnologías se verán impactadas. Algunos voceros gubernamentales de países desarrollados han mencionado que podrían ser utilizadas como palanca para salir de la crisis, por el contrario en este artículo se presenta el tema en el contexto de las tendencias a la concentración del capital.
\end{abstract}

Palabras clave: nanotecnología, crisis económica, capitalismo

\section{Nanotechnologies in the world crisis}

\begin{abstract}
The article discusses the development of nanotechnology in the context of the emergence of the global economic crisis. It starts by highlighting the key elements of a technological revolution: the technical characteristics, role in the accumulation of capital and the impact on social classes. After examining the place of technology in development, finally showing how the trends towards concentration of capital are involving nanotechnology, which can lead to a more unequal world than the one that exists today. Nanotechnology is the basis of the next industrial revolution which is already established. With the advent of the global economic crisis new technologies will also be impacted. Some government spokesmen have said that developed countries could be used as leverage to end the crisis, on the contrary in this article is stated the issue in the context of trends towards the concentration of capital.
\end{abstract}

Key words: nanotechnology, economic crisis, capitalism

Recibido: 10.04.2009

Aprobado: 10.6.2009

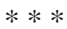

\footnotetext{
* Universidad Autónoma de Zacatecas, Zacatecas, México.E-mail: gfoladori@gmail.com

** Universidad Federal de Paraná, Curitiba, Brasil. E-mail: noela@ufpr.br
} 
En 2008 voceros gubernamentales de varios países desarrollados reconocieron que éstos habían entrado en recesión económica. Varios señalaron que las tecnologías de punta, y las nanotecnologías en particular, podrían ser un instrumento para salir de la crisis. Es sabido que la tecnología cumple un rol fundamental en el desarrollo, y que la historia del capitalismo está pautada por importantes revoluciones tecnológicas. Este artículo constituye un acercamiento al tema. Luego de analizar las características claves de las nanotecnologías como una revolución tecnológica, se muestra la principal tendencia a la concentración de la riqueza que estas tecnologías están manifestando, y cómo se puede inferir que la crisis económica mundial profundizará aún más aquella tendencia.

\section{¿Qué es una revolución tecnológica?}

Al analizar una revolución tecnológica hay que distinguir tres dimensiones que pueden ser aisladas teóricamente. En primer lugar, las características técnicas que hacen ventajosa a esa tecnología frente a las vigentes. La razón suele estar en productos que cumplen nuevas funciones, o que cumplen funciones que antes estaban distribuidas entre varios productos; en la utilización de nuevas fuentes de energía que hacen al producto más versátil o liviano, posible de ser utilizado en diversos y nuevos lugares, o tener una vida autónoma más duradera; en el cambio en los materiales del producto, que lo pueden hacer más liviano, adaptable a diversos ambientes, más resistente, o cumplir múltiples funciones; o, en varios de estos efectos combinados.

En segundo lugar, está el papel de la nueva tecnología en la acumulación de capital, lo cual explica el ritmo, nivel y extensión de la nueva revolución tecnológica en tiempo y espacio; y también la forma como incide en la división del trabajo. Aquí debe considerarse la versatilidad de la tecnología para ser aplicada a distintas ramas industriales; al contexto económico y comercial que permite la expansión geográfica; y, también, la trayectoria tecnológica a la cual la tecnología está amarrada, y que la hace más o menos dependiente del know how e infraestructura establecida; también el conjunto institucional de reglamentaciones es de importancia para la difusión de las nuevas tecnologías.

En tercer lugar es necesario analizar el impacto de la nueva tecnología en las clases sociales. Cómo estas son afectadas, y, en especial, el impacto sobre la clase trabajadora, así como las formas en que la nueva tecnología incide sobre la distribución geográfica de las clases.

La característica técnica de las nanotecnologías es la producción de nuevos materiales y el otorgamiento de nuevas funciones a los viejos. La manipulación de la materia a escala atómica, molecular y macromolecular, que es la base de las nanotecnologías, permite que la materia manifieste nuevas propiedades, diferentes a las conocidas en su tamaño bruto (RS\&RAE, 2004). Esto es posible debido a dos razones. Por un lado, por- 
que los nanomateriales tienen un área de superficie relativa mucho mayor si comparados con los mismos materiales en escala mayor. Esto hace que la reactividad química a nanoescala se potencie significativamente, al punto que algunos materiales no reactivos en su tamaño normal se vuelven reactivos. Por otro lado, porque al acercarse a la nanoescala las leyes cuánticas pasan a prevalecer sobre las de gravedad, afectando las propiedades ópticas, eléctricas, magnéticas, de resistencia, etc., de los materiales.

El carbono en su forma conocida como grafito, es blando y conductor eléctrico; en su forma de diamante, también conocido en la naturaleza, es el material más duro y no conduce la electricidad. Pero, los fullerenos de carbono, creados con nanotecnología, forman cristales de fullerita que, mezclados con elementos como rubidio y potasio, se convierten en superconductores. Por su parte, los nanotubos de carbono, también posibles de crear con nanotecnología, son muy rígidos, llegando a ser 100 veces más resistentes que el acero y, al mismo tiempo, 6 veces más livianos, siendo conductores o superconductores eléctricos.

En términos de la acumulación de capital, las nanotecnologías pueden considerarse el equivalente a conquistar un nuevo mundo, ya que el carácter dúctil de esta tecnología hace que pueda ser aplicada a prácticamente cualquier rama de la producción, con efectos devastadores sobre las antiguas tecnologías. Ya en 2008 productos de las nanotecnologías podían encontrarse en los más diversos sectores económicos, como la industria metalmecánica, aeroespacial, químico-farmacéutica, de energía, agro-alimenticia, informática, petroquímica, construcción, etc. Dadas las características disruptivas y el hecho de surgir en un mercado mundial altamente globalizado es previsible que la velocidad con que se imponga a nivel mundial, y la extensión que adquiera en términos geográficos, sea mucho mayor a cualquier revolución tecnológica anterior. En 2007 algunas consultoras en nanotecnología pronosticaban un mercado de productos que incorporaban nanotecnología de 3 mil millones de dólares para el 2014 o 2015, un crecimiento vertiginoso y expandido a nivel mundial (Científica 2007).

En términos del impacto de las nanotecnologías en la estructura de clases, es previsible el crecimiento del desempleo. Por un lado porque la multiplicación de funciones que pasan a realizar los productos de la nanotecnología reduce significativamente la cantidad de fuerza de trabajo necesaria, tanto al interior del proceso productivo como también en la manipulación, almacenamiento, transporte y comercialización de antiguos productos que desaparecerán del mercado. Además, las industrias que producen nanopartículas o nanoestructuras como materia prima son altamente tecnificadas, y no se espera que ocupen mucha fuerza de trabajo. Tampoco se espera mayor empleo en aquellas que incorporan nano-materias primas a sus procesos o productos. En realidad, como cualquier otra tecnología high-tech, se trata de una revolución tecnológica con poca incorporación de fuerza de trabajo. Por otro lado, porque una de las características de las nanotecnologías es que el producto se adapte al entorno mediante diversos 
tipos de sensores, se trata de una tendencia industrial histórica: a la mayor independencia frente a las contingencias ambientales y los recursos naturales. Con ello es previsible una nueva división espacial del trabajo, con los consecuentes desplazamientos y migraciones laborales.

\section{El papel de la tecnología en el desarrollo}

La revolución de las nanotecnologías ha revivido las tesis de los ciclos económicos largos de Kondratiev, y la crisis económica mundial se ha sumado para reivindicar su teoría. Según el célebre trabajo sobre los ciclos económicos del economista ruso Kondratiev (varias publicaciones entre 1922 y 1928), el desarrollo capitalista está pautado por ciclos largos de cerca de 50 a 60 años, que incluyen su fase de crecimiento y también de crisis. Cada una de estas fases dura de veinte a treinta años, y, en su conjunto el sistema tiende al equilibrio. ${ }^{1}$ Como Freeman \& Louçã señalan, Kondratiev no explicó los efectos de los ciclos en el proceso general e irreversible de acumulación de capital, que supone cambios estructurales significativos en la economía (Freeman \& Louçã, 2002, 80). Tampoco Kondratiev relacionó los ciclos económicos, que había detectado mediante análisis estadístico, con las luchas sociales y políticas, que también condicionan las fases y ciclos económicos. ${ }^{2}$ Además, es claro que si el capitalismo es una economía en acumulación, los ciclos, si existen, no tienen por que ocurrir con periodicidad regular, ni tener la misma duración, ni profundidad.

La mención a los ciclos Kondratiev, como se vinieron a llamar por Schumpeter, se hace necesaria hoy en día debido a que la revolución de las nanotecnologías augura una nueva era tecnológica, pero también debido a la irrupción de la crisis económica mundial. La escuela neoclásica en economía, que ha dominado el pensamiento económico de las últimas décadas, ha considerado a la tecnología como un factor externo dado en la función de producción; por ello esta concepción de poco sirve como armazón teórica para proyectos de desarrollo basados en nuevas tecnologías. Además, la teoría neoclásica siempre ha considerado a las crisis como un momento excepcional del desarrollo capitalista, como una enfermedad dentro de su evolución normal, o bien como un simple desajuste monetario que puede corregirse. Sin embargo, la realidad ha contradicho de tal forma esta visión que los economistas y políticos han buscado en el razonamiento mecánico de fases ascendentes y descendentes ligadas a revoluciones tecnológicas, y

\footnotetext{
${ }^{1}$ El primer ciclo u onda larga por él identificada era la que iba desde 1790s hasta 1810-17 como fase de crecimiento y de 1810-17 a 1844-51 como crisis. El segundo ciclo u onda larga iba de 1844-51 a 1870-75; y con su fase descendiente de 1870-75 a hasta 1890-95. El tercer ciclo por él analizado iba, en su fase ascendente desde 1890-95 hasta 1914-20, cuando comenzaba la fase descendiente (Freeman \& Louçã, 2002).

${ }^{2}$ Véase al respecto la crítica de Trotsky de 1923 en La curva del desarrollo capitalista (Trotsky, 2001).
} 
en el nombre de Kondratiev, una justificación para decir que el sistema capitalista es así, cíclico, y debemos acostumbrarnos a despegues y aterrizajes. Sin explicar, por cierto, las causas de la tendencia a la crisis más que por un supuesto agotamiento de la antigua tecnología, ni los efectos de esa crisis en las clases sociales, dando a entender que la crisis es mala para todos y los momentos de auge buenos para todos, ni las diferencias entre unas crisis y otras en extensión temporal y amplitud territorial; ni las causas del desarrollo tecnológico en el capitalismo.

Para entender por qué la historia del capitalismo ha estado puntuada por revoluciones tecnológicas es necesario considerar el papel de la tecnología en el desarrollo. La no consideración por parte del pensamiento neoclásico hegemónico de la tecnología y las crisis ha orientado a los políticos a apoyarse en los análisis schumpeterianos y neoschumpeterianos, y también marxistas, donde la tecnología es un elemento clave para explicar el desarrollo, y donde la relación entre las recesiones económicas y la introducción de nuevas tecnologías también es resaltada. De hecho todo el vocabulario de las políticas de ciencia y tecnología en cualquier país es schumpeteriano y neoschumpeteriano; términos como innovación, catching up, start ups, spinover y todo el bagaje conceptual de la "economía del conocimiento” tiene este origen teórico.

Schumpeter otorga a la competencia en el mercado el rol fundamental para la innovación. Los emprendedores, bajo la presión de la competencia, desarrollan alternativas tecnológicas que les ofrecen ventajas competitivas. Pero también Schumpeter relaciona esa tendencia intrínseca a la innovación con la necesidad de crédito para poder introducir las nuevas tecnologías. La innovación tendería a darse en los periodos de auge económico, ya que es allí que se pueden calcular los beneficios de los nuevos costos de la tecnología introducida, lo cual favorece el apoyo crediticio. Cuando existe recesión, la fluctuación de los precios hace difícil el cálculo de costos futuros, y con ello la innovación se vuelve más riesgosa y los créditos más difíciles.

La virtud del análisis schumpeteriano fue romper con la tradición neoclásica que consideraba el cambio técnico como algo externo. Para Schumpeter el cambio técnico es endógeno al proceso de desarrollo económico, y supone una tendencia a la acumulación de conocimientos y a la superación y evolución del sistema económico en un contexto de diferenciación y competencia. Pero, aunque la competencia en el mercado aparece como la fuerza que impulsa la innovación, Schumpeter no relacionó la innovación con los mecanismos de la ganancia a nivel del sector de producción en que se realiza y de la economía como un todo. La innovación aparece como un elemento clave en el proceso de desarrollo capitalista pero sin una explicación del rol que cumple en la acumulación de capital, y menos aún en las crisis económicas.

Medio siglo antes que se publicaran las principales obras de Schumpeter, Karl Marx había escrito mucho más profusamente sobre la 
relación entre el desarrollo tecnológico y la economía, y había establecido claros lineamientos para analizar la tecnología en relación con las crisis económicas.

La innovación tecnológica está basada, para Marx, en la tendencia de la competencia a incrementar permanentemente la productividad del trabajo. Aquellas empresas que aumentan la productividad en relación con las condiciones medias de su sector obtienen una ganancia extraordinaria, por arriba de la ganancia media. La competencia es la presión, y la ganancia extraordinaria el acicate para la innovación tecnológica; pero son las diferentes condiciones de producción las que explican las ventajas que se manifiestan en el mercado. Aunque la productividad del trabajo puede aumentarse exclusivamente a costas de una mayor intensificación de la explotación de la fuerza de trabajo (plusvalor absoluto), Marx explica que las luchas sociales y la difusión de la tecnología van homogeneizando esta posibilidad, llevando a que el aumento de la productividad por la vía de las mejoras tecnológicas (plusvalor relativo) se constituya en la norma de las sociedades capitalistas avanzadas (si se deja de lado el saqueo imperialista y las guerras, que son una fuente extraeconómica poderosa aún hoy en día ${ }^{3}$ ). Por ello, y en esto Schumpeter sigue a Marx, el cambio tecnológico es endógeno al sistema capitalista, puede ser explicado por su propia lógica interna, es un proceso acumulativo (evolutivo en términos schumpeterianos) y genera diferenciación entre aquellos que mejoran sus condiciones productivas por la innovación y quienes permanecen con las tecnologías anteriores.

Marx también explicó que el aumento de la productividad del trabajo se daba, como tendencia, por la incorporación de cada vez más tecnología por unidad de trabajo vivo, de esta manera la industria capitalista desplaza fuerza de trabajo. Posteriormente, la teoría económica neoclásica y keynesiana siempre argumentó que los trabajadores desplazados por la tecnología en una empresa eran captados por otras que se abrían. Esto, que nunca ha podido ser resuelto estadísticamente, no considera las implicaciones sociales, familiares y personales de aquellos que quedan sin trabajo y deben buscar nuevos empleos; pero de cualquier forma es un argumento cada vez menos creíble frente a las actuales altas tasas de desempleo y subempleo.

Una de las principales características de la nanotecnología es que sus productos tienden a concentrar utilidades o funciones, desplazando o haciendo obsoletas otras, con la consecuencia en una fuerte reducción de la ocupación. Este impacto será notable en algunas ramas de fuerza de trabajo especializada. Tomemos el caso de los trabajadores de los laboratorios de salud y enfermeros, aunque también doctores. Hoy en día sólo el 2\% de los productos con nanocomponentes pertenecen al área bio-médica, pero, según la consultora Científica éstos pasarán a ser el 80 por ciento del mercado en 2015 (Científica, 2007). Es de prever que este rápido aumento será comandado, en primera instancia, por aquellas investigaciones que permi-

${ }^{3}$ Véase a este respecto Harvey, 2007. 
tan abaratar los costos de la fuerza de trabajo especializada. Este es el caso de todo tipo de lab-on-a-chip, sean de aplicación externa al cuerpo o implantados. Estos dispositivos tienen la posibilidad de analizar en segundos cientos de bio-marcadores en gases, líquidos y tejidos, y enviar la información a una fuente externa de almacenamiento. Los laboratorios como los conocemos hoy en día quedarán reducidos en sus funciones y, con ello, su personal ocupado. Una segunda ola lo constituirán los productos dirigidos a enfermedades de clientes con alto poder adquisitivo en los países desarrollados (cáncer, etc.) con nanocápsulas inteligentes, capaces de alcanzar las células, los tejidos o los órganos enfermos y tratarlos in situ, evitando todos los daños colaterales que hoy en día conllevan los fármacos. Se trata de tecnologías que, más allá de la eficiencia, significarán un recorte sustancial en la fuerza de trabajo ocupada.

La potencialidad de desarrollar nuevos materiales y propiedades en los ya conocidos hace que un mismo producto adquiera funciones que antes eran desempeñadas por más de un producto. El pan TipTop de Austrália incorpora nanocápsulas (o microcápsulas) de Omega 3 (TipTop, s/f). De esta forma el pan cumple con la antigua función de alimento pero, también, con la función de suplemento alimenticio. Se estaría sumando en un producto dos utilidades o funciones, la del pan como alimento y la del suplemento alimenticio que ofrece el Omega 3. Lo que normalmente requiere empaque, comercialización y venta como actividades separadas en el pan y el suplemento, ahora se concentra en la misma mercancía. La empresa Industrial NanoTech, Inc. tiene patentado el nanocompuesto aislante Nansulate ${ }^{\circledR}$, que cumple diversas funciones, como el incrementar la capacidad de aislamiento térmico, al tiempo de ser anticorrosivo y funguicida (INI, s/f). Incorporan en un mismo producto funciones que antes cumplían varios productos diferentes. NanoSphere ${ }^{\circledR}$, un producto de Clariant and Schoeller, consiste en nanoesferas que pueden agregarse mediante un baño a los textiles, dando un efecto autolimpiante, repelente al agua, resistente a la abrasión y mejorando el desempeño al lavarse (Nanowerk News, 2008). En otros casos el nuevo producto desplaza al antiguo porque cumple la misma función de forma radicalmente mejor; como en el caso de los bloqueadores solares con nanotecnología, capaces de penetrar más profundamente la piel y bloquear los rayos ultravioletas. Aquí la misma función se cumple de manera más eficiente (BusinessWeek, 2005). Otro ejemplo es el aditivo Nanoprene, que la empresa LANXESS incorpora a la llanta de los automóviles para darle mayor durabilidad. En este caso las mismas materias primas trabajadas en nanoescala resultan en un producto más resistente (Nanotechnology Now, 2008). En el empaque de alimentos la nanotecnología también está presente. Un envase que protege el alimento de la humedad, de que se seque y del oxígeno, como lo hace el Durethan $\AA$, producido por Bayer (Bayer, s/f) le da mayor seguridad y vida útil al alimento, sustituyendo así los antiguos envases. En cualquiera de estos ejemplos, sea por sustitución de utilidades o funciones, sea por la mayor durabilidad, la consecuencia es una reducción previsible de la ocupación por producto producido. 
Es posible que nuevas ramas industriales surgirán y otras desaparecerán, pero siempre con un saldo negativo en la ocupación. Los textiles de origen vegetal, el hierro, el cobre, el café, el té, pueden llegar a reducirse como mercancías importadas por los países desarrollados; y, con ello, sectores enteros de la economía mundial como los conocemos hoy en día se verían trastocados (ETC Group, 2005a).

Por último, hay que considerar que cualquier reducción del desempleo provoca una reacción en cadena, porque los desempleados consumen menos, con lo cual se reduce la demanda para aquellas mercancías que son de consumo obrero, y, así, estas ramas se ven forzadas a incrementar la productividad para reducir costos, o bien reducir la producción y consecuentemente también el empleo; y así sucesivamente.

Uno de los grandes atinos de Marx fue prever que la ciencia se subordinaría a los intereses del capital. Desde principios de este siglo XXI en que se lanzan las políticas gubernamentales en nanotecnología en muchos países, hasta el 2005, más del 50 por ciento de las inversiones en Investigación y Desarrollo de nanotecnología provenía de financiamientos públicos. Es a partir del 2005 en que las inversiones privadas sobrepasan a las públicas (Científica, 2008). Esto significa que durante un par de décadas, si volvemos a mediados de los ochenta cuando comienzan las investigaciones en nanotecnología, la sociedad en su conjunto fue la que estuvo financiando el conocimiento y desarrollo de la nanotecnología. Pero, la forma en que las universidades y centros públicos de investigación se han enlazado con las empresas ha hecho que la orientación de la nanotecnología, una vez que sus productos comenzaban a estar prontos para llegar al mercado, se volcase directamente a la obtención de ganancias; muy lejos, de manifestarse en productos para la satisfacción de las necesidades más sentidas. Si tomamos como referencia la base de datos de productos de la nanotecnología que ya están en el mercado, elaborada por el Woodrow Wilson International Center for Scholars, vemos que la amplísima mayoría son productos de lujo. Podemos tomar como ejemplo la industria textil. En este sector la mayoría de los desarrollos de nanotecnología tienen relación con artículos deportivos, donde los costos son prohibitivos para los sectores populares, o bien se aplica la nanotecnología en la industria textil para tapizados de automóviles, en la industria de la construcción y otros fines que no son de consumo popular (WWICS, 2006). Otro argumento aún más contundente de esta relación entre investigación pública e intereses del capital está en el importante peso que la investigación militar viene teniendo en las investigaciones de nanotecnología en los Estados Unidos. Es bien sabido que en los Estados Unidos entre un cuarto y un tercio de los fondos públicos para nanotecnología han sido dirigidos directamente a investigaciones militares desde el 2004 (EOPUS, 2007). Científica resume así esta orientación hacia lo suntuario:

La mayor penetración de las nanotecnologías y también las tasas de crecimiento más altas se darán en las aplicaciones que no son sensi- 
bles al costo, tal como la médica, militar y textiles para deportes, donde el desempeño es normalmente más importante que el costo (Científica, 2008, 33).

Esta dependencia de la nanotecnología del financiamiento público, pero este último subsumido al servicio de la ganancia empresarial, muestra una revolución tecnológica planeada, muy lejos de la idea de la innovación desarrollada casi individualmente en la imagen de los emprendedores schumpeterianos.

Marx sostuvo, en su teoría de la ganancia, que la tecnología era utilizada por el capital para incrementar la productividad del trabajo con el fin de aumentar la tasa de ganancia. Esto es muy obvio, ya que ninguna tecnología se introduce si resulta en una ganancia menor a la que se obtiene con las viejas tecnologías. Sin embargo, los discursos sobre los beneficios de la nanotecnología poco hablan sobre su impacto en la ganancia, y sugieren que el motivo de su difusión es la mejor y mayor satisfacción de las necesidades sociales. Como todavía hay relativamente pocos productos de la nanotecnología en el mercado no es claro que sean competitivos con las viejas tecnologías en sus respectivas ramas. Sin embargo el hecho de que la mayoría de los que ya se comercializan sean productos suntuarios hace que sean menos sensibles al costo/beneficio, y en el caso de los productos destinados a la industria militar, el costo beneficio no existe como criterio. En cualquier caso el tipo de producto hasta ahora incorporado al mercado muestra que los motivos de la introducción de la nanotecnología poco tienen que ver con las necesidades sociales, y mucho con las ganancias empresariales.

Si las nanotecnologías se constituyen en la plataforma industrial de una nueva revolución tecnológica, expandiéndose a todas las ramas, y si, como argumentamos antes, exhiben una fuerte tendencia a la reducción de la ocupación, entonces se dará lo que también Marx previó: la caída tendencial de la tasa de ganancia en el largo plazo, debido a la reducción progresiva del trabajo vivo que es, en definitiva, el único que produce valor. De manera que, contra la idea de Kondratiev de que los ciclos del capital ocurren cada 50 o 60 años con fases de auge y de depresión, nos encontraremos con ciclos cada vez más cortos, donde las fases de depresión predominen en duración, espacio e intensidad. Sin embargo, en el corto plazo, y en el momento en que nos encontramos, las nanotecnologías pueden constituirse en una tabla de salvación para muchos capitales. Marx fue enfático en afirmar que la crisis es uno de los mecanismos más eficientes en recuperar la caída de la tasa de ganancia del capital. En la medida en que se esfuman miles de millones de dólares de acciones de la empresas, se reduce el valor del capital constante movilizado por el capital, de manera que la proporción de las ganancias en relación al capital invertido, es decir, la tasa de ganancia, vuelve a aumentar y pone a las nuevas tecnologías en un contexto ideal para convertirse en caballito de batalla del capital para retomar la hegemonía social que la crisis le hizo perder. 


\section{La tendencia a la concentración del capital de las nanotecnologías}

Desde los años ochenta las investigaciones de la materia en tamaño nano comenzaban a tener presencia en los laboratorios científicos. Pero, no fue sino hasta el lanzamiento de la National Nanotechnology Initiative de los Estados Unidos en el 2000, cuando los apoyos financieros gubernamentales crecen significativamente a nivel mundial, y las nanotecnologías pasan a ser contempladas como una de las áreas de desarrollo científicotecnológico más promisorias. Para el quiebre de siglos los Estados Unidos eran, y continuaron siendo por varios años, el primer país en los índices de competitividad internacional. Sin embargo, los analistas ya advertían, desde mediados de la década del noventa, que la brecha de competitividad se venía reduciendo significativamente. En esos momentos el paradigma de la Economía del Conocimiento como clave del desarrollo estaba en pleno auge, y el informe del Banco Mundial de 1998-1999 lleva el subtítulo Knowledge for Development, y estaba dedicado a la brecha de conocimiento entre países ricos y pobres. Los indicadores de desempeño en ciencia y tecnología mostraban que los Estados Unidos estaban perdiendo competitividad relativa frente a países asiáticos y a Europa. Los estudiantes sud coreanos, chinos y taiwaneses que hacían sus doctorados en Estados Unidos cayeron en más de un $20 \%$ durante la década de los noventa, prefiriendo crecientemente sus países de origen. Estados Unidos fue sobrepasado por Europa Occidental desde 1996 en artículos científicos publicados. En patentes, a pesar que los Estados Unidos estaban a la cabeza, el acelerado crecimiento asiático visualizaba una equiparación a finales de la primera década del siglo XXI; y lo mismo se preveía en el caso de las inversiones en ciencia y tecnología (TFFAI, 2006). El resultado general va a ser la implementación, por parte de los Estados Unidos, de una política de investigación y desarrollo que apuesta a la nanotecnología como instrumento para mantener la hegemonía mundial en materia de competitividad económica. Así, no debe sorprender que el mensaje de la Casa Blanca en apoyo a la nanotecnología llevase por subtítulo “Leading to the Next Industrial Revolution”, mostrando la importancia que la política estadounidense visualizaba en la nanotecnología. ${ }^{4}$ Cuando se lanza la Iniciativa Nacional ya existían las condiciones institucionales y empresariales preparadas para el rápido desarrollo que cristalizó en pocos años. De hecho, la nanotecnología en cuanto desarrollo científico-técnico se realiza en momentos de auge del ciclo económico capitalista, durante la segunda mitad de los ochenta y toda la década de los noventa. Aunque es importante mencionar que en todo este periodo el grueso del financiamiento fue público, sólo a partir de 2005 las inversiones privadas comienzan a pasar las inversiones públicas (Científica, 2008).

¿Pero, qué pasará con la nanotecnología una vez que se manifiesta la crisis mundial en 2008? Algunos políticos y científicos ya están previen-

\footnotetext{
${ }^{4}$ Los planes para el desarrollo de una iniciativa gubernamental de apoyo a la nanotecnología en Estados Unidos comienzan en 1996 (http://www.nano.gov/html/about/history.html).
} 
do que estas tecnologías pueden ser la salida de la crisis. ${ }^{5}$ Sin embargo, aún cuando la nanotecnología pudiera llegar a tener este papel de salvataje de las ganancias capitalistas, hay algunas tendencias que claramente se verán reforzadas. La más importante es la concentración y centralización del capital. Esta, que es una tendencia permanente del sistema capitalista, se ve reforzada en momentos de crisis, cuando los pequeños y medios capitales, salvo excepciones, no pueden resistir y terminan vendiendo o asociándose con las grandes corporaciones transnacionales.

La crisis devalúan los bienes de muchas empresas. Bajo estas circunstancias el capital de riesgo deja de ser rentable, o, en el mejor de los casos no confía en inversiones inciertas. Hay pocas posibilidades de obtener crédito o de atraer capital para las pequeñas empresas de manera de permitirles entrar al mercado con algún grado de éxito. Varios consultores coinciden en este diagnóstico.

“... los altos factores de riesgo combinado con los altos retornos por la inversión que el capital de riesgo demanda significa que el apoyo de este capital a las empresas que recién comienzan en nanotecnología son cuando menos débiles” (Harper, 2008, 2-3).

\section{Y, también,}

Esto plantea problemas sustanciales para las empresas de nanotecnología. Primero, ahora será más difícil recaudar capital, y aún más difícil vender nuevos productos. Recaudar capital es importante para firmas de alta tecnología, tal como las empresas de nanotecnología, ya que es probable que se requieran muchos recursos antes que la empresa llegue a ser rentable.

El capital de riesgo es probablemente el más común en las empresas de alta tecnología. Allí las empresas de nanotecnología pueden encontrar una fuente de fondos para el desarrollo. La desventaja, sin embargo, es que las firmas de capital de riesgo, u otro tipo de empresas privadas como los ángeles empresariales, requieren una creciente tasa de retorno sobre sus inversiones, de manera de compensar los altos riesgos asociados. Agregado al tradicional volumen de riesgo en las firmas de alta tecnología, las actuales advertencias de suspensión de liquidez en los mercados financieros y el riesgo del crédito mencionado arriba, aumentan la tasa de préstamos del dinero, y, consecuentemente, las tasas de retorno. Estas tasas pueden ser prohibitivas para algunos empresarios de nanotecnología, que naturalmente también quieren una buena parcela de retorno por su riesgoso esfuerzo (Petratos, s/f).

\footnotetext{
${ }^{5}$ Véanse, por ejemplo, las declaraciones del vicepresidente de Rusia (Prensa Latina, 2008), del ministro de Ciencia y Tecnología de Sri Lanka (Peiris, 2008), o de un asesor del departamento de ciencia y tecnología del gobierno de la India (Gupta Ray, 2009).
} 
La tendencia a la concentración del capital ya se venía presentando en las nanotecnologías desde los noventa. Veamos, por ejemplo, información relacionada con el porcentaje de inversión pública en nanotecnología. La consultora Científica provee los siguientes datos para los primeros diez países (Cuadro 1).

\section{Cuadro 1}

Inversiones en Investigación y Desarrollo de nanotecnología. Estimaciones para 2008

\begin{tabular}{|l|c|c|c|c|}
\hline País & $\begin{array}{c}\text { A } \\
\text { Clasificación }\end{array}$ & $\begin{array}{c}\text { B } \\
\text { Inversiones } \\
\text { en dólares de } \\
\text { Estados Unidos } \\
\text { (millones) } \\
\text { Estimaciones } \\
\text { para 2008 }\end{array}$ & $\begin{array}{c}\text { C } \\
\text { Inversiones } \\
\text { en dólares de } \\
\text { Estados Unidos } \\
\text { (millones) } \\
\text { corregidos por la } \\
\text { capacidad de } \\
\text { compra por país } \\
\text { Estimaciones } \\
\text { para 2008 }\end{array}$ & $\begin{array}{c}\text { D } \\
\text { Clasificación }\end{array}$ \\
\hline Mundo & 1 & 7,849 & 2,787 & 1 \\
\hline UE 25 & 2 & 1,821 & 1,821 & 4 \\
\hline USA & 3 & 1,128 & 995 & 5 \\
\hline Japón & 4 & 1,076 & 2,107 & 2 \\
\hline Rusia & 5 & 541 & 411 & 7 \\
\hline Alemania & 6 & 510 & 2,034 & 3 \\
\hline China & 7 & 350 & 440 & 6 \\
\hline Coréa del Sur & 10 & 184 & 141 & 10 \\
\hline UK & 8 & 97 & 175 & 9 \\
\hline Taiwan & 9 & 50 & 213 & \\
\hline India & & & & \\
\hline
\end{tabular}

Fuente: basado en Cientifica, 2008. The Nanotechnology Opportunity Report. 3rd Edition. http://cientifica.eu/index.php?option=com_virtuemart\&page=shop.product_details\&flypage =shop.flypage\&product_id=34

El cuadro 1 anterior muestra a los primeros diez jugadores en nanotecnología, según las inversiones públicas para Investigación y Desarrollo. Podemos observar que los únicos países en desarrollo del cuadro son China e India, ambos países con enormes Productos Internos. El reporte ofrecido por Científica argumenta que la columna B no refleja el papel de cada uno de esos países en el desarrollo de la nanotecnología. Entonces ofrece una clasificación diferente, basada en la capacidad de compra de la 
moneda local en cada país (columna C). Este último cuadro muestra que un investigador de Alemania, por ejemplo, cuesta más que uno de los Estados Unidos; y uno de la China o India es significativamente más barato (Científica, 2008, 37). Por esta razón, China e India son clasificados más altos usando este último criterio. Sin embargo, el hecho de que solo países desarrollados o algunos pocos en desarrollo con un producto anual enorme son los primeros en la carrera de la nanotecnología no es un resultado arbitrario de la voluntad de los gobiernos, pero significa que la revolución tecnológica demanda altos volúmenes de capital. Esta tendencia a la concentración de la nanotecnología en los países más desarrollados, y algunos en desarrollo pero con economías de escala continental, se acrecentará aún más con la crisis mundial desatada en 2008.

Otro indicador de la concentración de la riqueza es el monopolio sobre las patentes. Según datos recopilados por la OEI en 2009, entre 2000 y 2007 la Organización Mundial de la Propiedad Intelectual tenía registradas 75720 patentes participantes del Tratado de Cooperación en materia de Patentes

\section{Cuadro 2}

Gráfico 49. Titulares de patentes en nanotecnologia

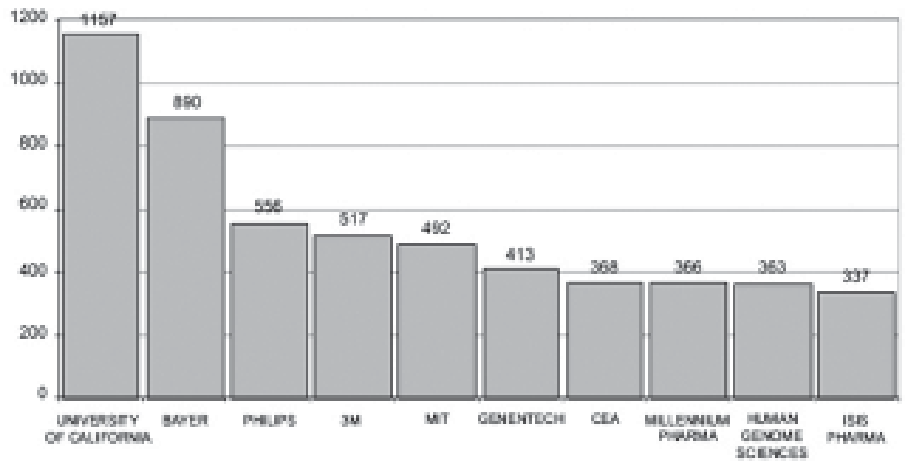

Fuente: OEI, 2009.

Podemos observar la concentración de patentes en corporaciones transnacionales y grandes universidades estadounidenses que trabajan en estrecho contacto con empresas y el Departamento de Defensa de los Estados Unidos, como es el caso de la Universidad de California y el MIT.

Otro indicador de la concentración de la riqueza es la producción de nanopartículas y nanoestructuras como materia prima para la industria. El reporte de Científica es también claro a este respecto. Muestra que luego de unos años de desarrollo de nanopartículas y nanoestructuras las grandes corporaciones químicas transnacionales han controlado el mercado. La ex- 
plicación es que solo empresas como BASF, BAYER o DOWN Chemicals pueden ofrecer nanopartículas y nanoestructuras con la calidad y en el volumen que la demanda exige.

"Hace cinco años, si usted quería nanomateriales, debía lidiar con unos pocos tipos en un garaje, o con un profesor y algunos estudiantes de graduación consiguiendo un poco de circulante por ese lado. Si ahora necesita el mismo material puede comprarlo de varias industrias químicas bien establecidas. La clave es que estos grandes proveedores tienen procedimientos de control de calidad rigurosos. Eso significa que si Boeing quiere usar un nuevo compuesto basado en nanopartículas, tendrán certeza que podrán comprar la misma cosa la semana próxima, el año próximo o dentro de diez años, y que empresas como BASF aún estarán en el negocio el mes próximo. Esta habilidad de comprar amplias cantidades de materiales bien caracterizados es lo que llevará a la nanotecnología al mercado. Mientras que mis cifras indican que la tasa de crecimiento de la industria de nanomateriales muestra una saludable tasa compuesta de crecimiento anual de entre 20 y 30 por ciento para los próximos cinco años, el crecimiento realmente explosivo será en la aplicación de tales materiales" (Científica, 2008, 28).

Y, si tomamos el caso de los nanocomponentes que se utilizan como materia prima en muchas industrias (nanotubos de carbono, nanocables, quantum dots, fullerenos, diversas partículas) podemos ver, en la base de datos de Nanowerk, que casi el 50 por ciento de las empresas que suministran materias primas están localizadas en Estados Unidos (146 en un total de 305 para marzo 3 de 2009) (Nanowerk-Database, s/f). Claro que la base de datos de Nanowerk no pretende ser exhaustiva, y no considera toda información que no esté en la Web en inglés, pero la recopilación de información periodística y académica durante varios años hace de ésta una fuente muy indicativa del movimiento general de la nanotecnología.

Otro ejemplo que ilustra la concentración de la riqueza en nanotecnología es lo que está ocurriendo con la industria textil. Es sabido que en los noventa esta industria estableció la mayoría de sus líneas de producción en el Sudeste asiático. Sin embargo, la mayoría de las nanopartículas o nanocomponentes necesarios para ser aplicados a los textiles están siendo desarrollados en los Estados Unidos (Científica, 2008). Esto significa que, si esta tendencia continúa, los Estados Unidos controlarán el grueso de la industria nano-textil aunque la mayoría de las líneas de producción estén basadas en Asia. Asia tendrá que comprar los necesarios nanocomponentes a los Estados Unidos, una reestructuración del mercado de los nano-textiles que concentrará aún más el poder y la riqueza.

Claro está que podrá haber casos en que países pobres, sectores o pequeñas empresas salgan victoriosas de la depresión económica, pero esto no será la norma. Un ejemplo sectorial podría ser el del aumento de la demanda de litio. La nueva generación de automóviles eléctricos incrementará sustancialmente la demanda de litio para sus baterías, ya que 
este mineral permitiría superar el problema del limitado tiempo de autonomía de las baterías y el excesivo tiempo de carga (The Economist, 2009). Como hoy en día el $50 \%$ de las reservas de menor costo de este mineral están en Bolivia, esta tendencia de la nanotecnología colocará a un país en desarrollo en una situación privilegiada (Romero, 2009).

Este efecto tiene implicaciones para la inequidad y el subdesarrollo. La concentración del poder de la nanotecnología en manos de unas pocas corporaciones transnacionales con sede en los países desarrollados va de la mano con la inequidad. Bajo este esquema no hay otro resultado que la concentración de la riqueza, aún cuando esto signifique incremento en la inequidad, o en el número de desposeídos, sean países, empresas, clases o personas. El problema de la inequidad, que incluye desempleo y pobreza, no es responsabilidad de una entidad en particular, ni de una institución, ni de muchas organizaciones. Es el resultado final de un proceso que estimula la competencia bajo condiciones desiguales.

\section{Conclusiones}

La revolución de las nanotecnologías, si llega a concretarse como es la esperanza de muchos sectores como una nueva revolución industrial, no será la panacea para la mayoría de la población mundial. Las tendencias económicas dentro de las cuales estas tecnologías se han desarrollado, de fuerte enlazamiento con las corporaciones transnacionales, de importante presencia en la industria militar, de fuerte concentración de la riqueza, hacen de estas tecnologías un instrumento del capital para aumentar la productividad del trabajo y, consecuentemente, las ganancias. Esto no traerá beneficios automáticos para los trabajadores y los pobres, como dicen muchos tecnólogos. Por el contrario, sólo mediante las luchas sociales podrán los más necesitados obtener algunas ventajas de estas tecnologías.

Pero si alguna esperanza podían traer las nanotecnologías en términos de mejoras en áreas como la salud y el medio ambiente, la manifestación de la crisis mundial en 2008 va, seguramente, a concentrar aún más la orientación de la investigación a los sectores con mayor poder adquisitivo. Además, si muchos países tenían en sus agendas a la nanotecnología como instrumento de desarrollo, estas posibilidades se verán reducidas por la crisis, y esto será más grave en los países menos desarrollados. Si bien es cierto que desde 2005 más del 50 por ciento de las inversiones en investigación y desarrollo a nivel mundial provienen de la empresa privada, esto no es homogéneo, en los países menos desarrollados siguen siendo los fondos públicos los que financian la nanotecnología; de manera que la recesión económica, con la reducción de la producción y del empleo implicará recaudaciones menores y, consecuentemente, una reducción de los presupuestos para educación e investigación y desarrollo. 


\section{Bibliografía}

Bayer (s/f), Securely wrapped. Nanoparticles make Durethan ${ }^{\circledR}$ films airtight and glossy. http://www.research.bayer.com/edition_15/15_polyamides.pdfx Last visited March 12, 2008.

BusinessWeek (2005), Nano, Nano, On the Wall.... L’Oreal and others are betting big on products with microparticles, BusinessWeek, December 12. http://www.businessweek.com/magazine/content/05_50/b3963100.htm Last visited May 12, 2007.

Científica (2007a), Half Way to the Trillion-Dollar Market? A Critical Review of the Diffusion of Nanotechnologies. http:/www.cientifica.eu/ index.php?option=com_frontpage\&Itemid=1 Last visited April 24, 2007.

Idem (2007b), The Nanotechnology Opportunity Report. 3rd Edition. http:/ / cientifica.eu/index.php?option=com_virtuemart \&page = shop.product_details\&flypage=shop.flypage\&product_id=34 Last visited February 16, 2009.

EOPUS (Executive Office of the President of the United Status) (2005), The National Nanotechnology Initiative. Research and Development Leading to a Revolution in Technology and Industry. Supplement to the President's 2006 Budget. http://www.nano.gov/NNI_06Budget.pdf Last visited October 18, 2006.

ETC Group (2005a), The potential impacts of nano-scale technologies on commodity markets: The implications for commodity dependent Developing countries. South Centre Trade Research Papers, 4. http://www. southcentre.org/publications/researchpapers/ResearchPapers4.pdf Last visited June 09, 2006.

Idem (2005b), Nanotech's “Second Nature” Patents: Implications for the Global South. March/April and May/June-2005. Communiqués No. 87 and 88. http://www.etcgroup.org/documents/Com8788SpecialPNanoMarJun05ENG.pdf Last visited September 1, 2006.

Freeman, Chris \& Louçã, Francisco (2002), As Time Goes By. New York: Oxford University Press.

Gupta Ray, Shashwat. (2009), Nanotech, a big way to beat recession. [News about the declarations of Rajiv Sharma, advisor at international cooperation division of Department of Science and Technology, Government of India]. Sakaal Times, March 11, 2009. http://www.sakaaltimes.com:80/2009/03/ 11115048/NANOTECH-A-BIG-WAY-TO-BEAT-RE.html Last visited March 12, 2009.

Harper, Tim (2009), Nanotechnologies in 2009. Creative Destruction or Credit Crunch? White Paper. Científica Ltd. http://www.cientifica.eu/files/ 
Whitepapers/Nanotechnologies\%20in\%202009.pdf Last visited February 16, 2009.

Harvey, David (2007), Neoliberalism as creative destruction. Annals of the American Academy of Political Social Science. 610, 21.

INI (Industrial NanoTech, Inc.) (s/f), NanoSphere®. http://www.industrialnanotech.com/index.html Last visited March 06, 2009.

Nanotechnology Now (2008 January), Tire service life extended. Nanotechnology Now. http://www.nanotech-now.com/ news.cgi?story_id=27514 Last visited March 12, 2009.

Nanowerk News (2008). NanoSphere At The Cutting Edge. Nanowerk News. May 15, 2008. http://www.nanowerk.com/news/newsid=5727.php Last visited March 06, 2009.

Nanowerk-Database (s/f), Nanotechnology Nanomaterial Suppliers. Database. http://www.nanowerk.com/nanotechnology/nanomaterial/ nanomatmatrix.php Last visited March 5, 2009.

OEI (Organización de Estados Iberoamericanos para la Educación, la Ciencia y la Cultura) (2009 febrero 2), La nanotecnología en Iberoamérica. Situación actual y tendencias. Informe del Observatorio Iberoamericano de Ciencia, Tecnología e Innovación del Centro de Altos Estudios Universitarios de la OEI. Retrieved from http://www.oei.es/salactsi/nano.pdf Last visited March 10, 2009.

Peiris, Manjari (2008), State sector has to play an important role in riding present financial crisis urges Professor Tissa Vitarana. Asiantribune.com November 21, 2008. http://www.asiantribune.com/?q=node/14314 Last visited January 10, 2009.

Petratos, Phytágoras (s/f), How the current economic crisis will affect nano. Nano http://www.nanomagazine.co.uk/readComment.php?id=3 Last visited March 10, 2009.

Prensa Latina (2008), Russia: Nanotechnology against world crisis. http:/ /www.plenglish.com/article.asp?ID=\%7B77F977C5-D097-4560-93EE0075ED621937\%7D)\&language=EN Last visited February 04, 2009.

Romero, Simon (2009), In Bolivia, untapped bounty meets nationalism. The New York Times, February 03, 2009.

RS\&RAE (Royal Society \& The Royal Academy of Engineering) (2004), Nanoscience and nanotechnologies: opportunities and uncertainties. London: The Royal Society \& The Royal Academy of Engineering. www.royalsoc.ac.uk/policy www.raeng.org.uk Last visited February 12, 2005. 
TFFAI (The Task Force on the Future of American Innovation) (2006), The Knowledge Economy: Is the United States losing its competitive edge? http://www.futureofinnovation.org/PDF/Benchmarks.pdf Last visited March 15, 2009.

The Economist (2009 March 12), Batteries now included http:// www.economist.com:80/science/displaystory.cfm?story_id=13277371 Last visited March 19, 2009.

Tip Top (s/f), Tip Top ${ }^{\circledR}$ UP $^{\text {TM }}$. www.tiptop.com.au Last visited March 06, 2009.

Trotsky, León (2001), La curva del desarrollo capitalista, Razón y Revolución, 7. http://www.razonyrevolucion.org.ar/textos/revryr/economia/ ryr7Trotsky.pdf Last visited March 10, 2009.

WWICS (Woodrow Wilson International Center for Scholars) (2006), A Nanotechnology Consumers Product Inventory. Project on Emerging Nanotechnologies. www.nanotechproject.org/index.php?id=44 Last visited March 11, 2006. 